Pedagogía y Saberes No. 51

Universidad Pedagógica Nacional

Facultad de Educación. 2019. pp. 23-32

\title{
La construcción del rol docente desde una mirada renovadora de la lectura y la escritura*
}

\section{Artículo de investigación}

The Construction of the Teaching Role from

a Renewing Look of Reading and Writing A construção do papel docente de um olhar renovador de leitura e escritura

Pablo Enrique Arellano García**

Para citar este artículo:

Arellano, P. (2019). La construcción del rol docente desde una mirada renovadora de la lectura y la escritura. Pedagogía y Saberes, 51, 23-32

Esta investigación formó parte del doctorado en Lingüística que desarrollamos en la Universidad de Los Andes de Venezuela.

** Asesor académico de la Universidad Nacional Abierta de Venezuela. Doctor en Lingüística de la Universidad de Los Andes de Venezuela e investigador del Grupo Formación Docente.

Correo electrónico: icad.pabloarellano@gmail.com

Código ORCID: 0000-0001-8387-8211 


\title{
Resumen
}

El artículo presenta algunas reflexiones derivadas del proyecto de investigación Estudio del tópico en los proyectos pedagógicos de aula sistematizados por docentes de Educación Básica. Allí se reflexiona sobre el trabajo de varios años compartidos con otros docentes en ejercicio y con estudiantes de pregrado y posgrado que permiten un análisis constante de la práctica pedagógica. Se abordan tres aspectos: 1) la transformación docente. Asunto en el que se reconoce la existencia de los saberes previos, que desmitifica muchas de las viejas formas conductistas de entender la práctica pedagógica. 2) El papel de la lectura y la escritura en la construcción de un nuevo rol docente. Aspecto que permite a los docentes leer experiencias escritas por otros y que les ayuden a ampliar su visión del mundo, incidiendo en su didáctica y en la reconstrucción de los esquemas propios como lector y escritor. 3) La relación escuela-universidad en el proceso de cambio de las prácticas pedagógicas de los docentes. Dimensión que es necesario revisar a la luz de lo que significa intelectual, social y afectivamente el acto lector y escritor. Finalmente se presentan algunas consideraciones sobre el papel de la lectura y la escritura en las nuevas formas de pensar el trabajo docente.

Palabras clave

rol docente; proyectos; lectura; escritura

\begin{abstract}
The article presents some reflections derived from the research project Study of the topic in classroom pedagogical projects systematized by teachers of Basic Education. There it reflects on the work of several years shared with other teachers in exercise and with undergraduate and postgraduate students that allow a constant analysis of the pedagogical practice. Three aspects are addressed: 1) teacher transformation. This issue recognizes the existence of previous knowledge, which demystifies many of the old behavioral forms of understanding pedagogical practice. 2) The role of reading and writing in the construction of a new teaching role. An aspect that allows teachers to read experiences written by others and to help them to expand their vision of the world, by influencing their didactics and the reconstruction of their own schemes as a reader and writer. 3) The school-university relationship in the process of change teacher pedagogical practices. A dimension that needs to be reviewed in the light of what the reader and writer act mean intellectually, socially and affectively. Finally, it's presents some considerations on the role of reading and writing in new ways of thinking teaching work.
\end{abstract}

\section{Keywords}

teaching role; projects; reading; writing

\section{Resumo}

0 artigo apresenta algumas reflexões derivadas do projeto de pesquisa Estudo do tópico nos projetos pedagógicos de aula sistematizados por docentes de Ensino Fundamental. Reflete-se sobre o trabalho de vários anos compartilhado com outros professores em exercício e com alunos de graduação e pós-graduação que permitem uma análise constante da prática pedagógica. Três aspectos são abordados: 1) transformação do professor. Assunto no qual se reconhece a existência de conhecimentos prévios, o que desmistifica muitas das antigas formas de compreender a prática pedagógica. 2) 0 papel da leitura e da escrita na construção de um novo papel docente. Aspecto que permite aos professores ler experiências escritas por outros e que os ajudam a ampliar sua visão de mundo, modificando a sua didática e a reconstrução de seus próprios esquemas como leitor e escritor. 3) A relação escola-universidade no processo de mudança das práticas pedagógicas dos professores. Dimensão que é necessário rever à luz do que significa intelectualmente, socialmente e emocionalmente o ato de ler e escrever. Finalmente, apresentam-se algumas considerações sobre o papel da leitura e a escrita nas novas formas de pensar o trabalho docente.

\section{Palavras-chave}

papel de professor; projetos; leitura; escrita 


\section{Introducción}

Las reflexiones que siguen son producto del trabajo de varios años compartidos con otros docentes en ejercicio y estudiantes de posgrado. Asimismo, constituyen uno de los resultados del proyecto de investigación ya culminado: Estudio del tópico en los proyectos pedagógicos de aula sistematizados por docentes de Educación Básica. ${ }^{1}$ Estas ideas también se comentan a partir de la experiencia como maestro de niños, como profesor universitario y formador de formadores.

Entonces, producto del trabajo compartido, intentando desarrollar experiencias didácticas desde una óptica constructivista para la enseñanza de la lengua escrita en Educación Básica, he llegado a plantearme, entre muchos otros, los siguientes interrogantes, sobre los cuales gira esta disertación:

- ¿En qué contexto se desarrolla la transformación del docente?

- ¿Cómo la posibilidad en la construcción del conocimiento, producto de la investigación, redimensiona la perspectiva de la lectura y la escritura y obliga al docente a revisar su rol en esta dirección?

- ¿Cuál es el papel de la universidad en el proceso de cambio en las prácticas pedagógicas?

\section{La transformación docente: ¿por qué?, ¿cómo? y ¿para qué?}

En un mundo de cambios donde los medios de comunicación son cada vez más arrolladores, con los cuales el niño pasa la mayor parte de su tiempo y donde se muestra lo que sucede día a día en el mundo, sin duda es imperativo un replanteo en las prácticas pedagógicas para dejar el viejo sistema de dar clase. Este replanteo debe pasar por abandonar las pruebas escritas y el uso del tablero y la tiza, para incorporar situaciones didácticas que respeten el proceso de los aprendices.

Como dicen Dubois (2011) y Salas (2012), cuando un docente está dispuesto a transformar su forma de enseñar, lo primero que se pregunta es cómo hacerlo. El análisis y la discusión junto a otros docentes han llevado a pensar que no hay un interruptor único que encienda el bombillo del educador para que se apropie de un nuevo esquema didáctico. Sin embargo, a lo largo de varios años hemos ido construyendo algunos planteamientos interesantes, los cuales no producen

1 Esta investigación se desarrolló con docentes, a partir de talleres de escritura sobre sus prácticas pedagógicas para luego publicar sus trabajos en la revista Maestros Hoy. ningún efecto, si el cambio hacia nuevos paradigmas no se inicia de forma interna en cada uno de los docentes; pues, es solo desde la experiencia de cada uno de los actores reales que puede crearse un espacio innovador en el aula, la escuela y la comunidad. Como dice Dubois (1995), la formación permanente es una cuestión de elección individual que corresponde a la persona y al profesional que es el maestro.

Afirma que hay algunos elementos salidos de la experiencia que ayudan a la formación del docente en la búsqueda de una práctica pedagógica renovadora y que coinciden con las ideas de Díaz (2016):

- Primero, por supuesto, es tener la disposición para asumir el compromiso, sin este indicador todo lo demás carece de sentido.

- Luego, es necesario incorporarse a un circuito de capacitación con instancias que respeten los saberes de cada uno y permitan la reflexión permanente.

- Revisar de forma constante los aspectos teóricos a la luz de lo que sucede en el aula de clase. Esto llevaría al docente a convertirse en investigador de su propia práctica.

- Interactuar con otros compañeros que estén transitando el mismo camino, alienta el enriquecimiento mutuo.

- Conformar equipos de estudio en cada escuela y compartir las experiencias intraescolares con otros maestros.

- Tratar de permanecer en el proceso de formación y desarrollar investigaciones a la luz de su propia práctica.

Probablemente, son muchos los elementos que contribuyen con una manera distinta de entender la praxis en un paradigma interaccionista, pero son estos los que nos han posibilitado el acceso a un mundo de verdaderos cambios en nuestra manera de enseñar. Para ello, es fundamental contar con espacios de formación y actualización permanentes, y participar de modo activo en ellos; lo que llamaríamos entrar a un circuito de formación permanente. Durante estos espacios se analiza el proceso de aprendizaje y el papel activo, tanto del maestro como del alumno, como sujetos cognoscentes. Aquí encontramos que las proposiciones de Piaget citadas por Camps (2001) cobran cada día más fuerza, pues la adquisición de nuevos conocimientos se da con mayor significación cuando lo que se asimila puede contrastarse con lo que se tiene en el cerebro y así se da paso a nuevas formas de adaptación. 
En este contexto, nos encontramos con las ideas de Castedo (2010) con quien coincidimos, en tanto pensamos que la enseñanza se va cargando de un significado nuevo cuando se reconoce la existencia de los saberes previos, que desmitifica muchas de las viejas formas conductistas de entender la práctica pedagógica. Tal reconocimiento lleva al docente a observar los hechos del aula y a escribir su propia historia para compartirla con otros docentes, es decir, a hacer investigación acción. Desde nuestra práctica, esto nos ha permitido desplegar actitudes distintas en cuanto a las situaciones didácticas que deben propiciarse en las aulas de clase, dándole a la lectura y a la escritura el espacio que merecen para el desarrollo de todos los demás aprendizajes.

\section{El papel de la lectura y la escritura en la construcción de un nuevo rol docente}

Si la labor principal de los educadores es enseñar (con particular atención a los que se desempeñan en los grados inferiores y medios) para lograr que sus alumnos aprendan a leer y escribir, y si la didáctica de la lectura y la escritura se ocupa de los problemas que los docentes necesitan resolver para comunicar estas prácticas a sus alumnos en contextos específicos de enseñanza, entonces, el estudio didáctico de estos objetos de conocimiento debería ocupar el lugar central en la formación docente. Es decir, como refiere Silva (2014), el docente debe ser un constructor permanente de la lectura y la escritura, para entender lo que sucede durante las clases. En tal sentido, los profesores deben interesarse en estos objetos y verlos como fuente de interés para la investigación que permita a los alumnos otorgar importancia a los actos de lectura y escritura. De hecho, Jiménez (2018) acota que algunos estudios han mostrado que la escritura más frecuente en las aulas de clase es la toma de apuntes y que una amplia mayoría de los alumnos la ponen en práctica en las distintas materias que cursan; además, si esto lo hacen con un docente entusiasmado, el aprendizaje surgirá de manera significativa.

En este camino, la lectura y la escritura -en nuestra experiencia- se convirtieron en las herramientas fundamentales del trabajo pedagógico de todos, pues creamos el taller Expresión escrita de experiencias pedagógicas, en el cual analizamos, entre otros aspectos, las ideas de Cassany (2003) quien plantea que al escribir se utiliza el lenguaje con una finalidad específica, se alcanzan objetivos y se adquiere la capacidad de darle significación a las palabras en cada contexto según la intención del autor. Como dicen Gutiérrez y
Molina (2017), la escritura es vista como un proceso que requiere articular la elaboración y posterior redacción de pensamientos, trabajar tanto con las ideas como con las palabras; es un instrumento de aprendizaje, ya que, al escribir, se está aprendiendo tanto del objeto de conocimiento que se escribe como del proceso de escritura.

El mismo Cassany (1999) refiere que la escritura es una manifestación de la actividad lingüística con un objetivo determinado, es una forma de usar el lenguaje en pos de estos objetivos y en algunas situaciones las intenciones modifican el significado convencional de las expresiones. Escribir no requiere solo de la habilidad de redactar, sino también de la lectura y la comprensión lectora: releemos los borradores que vamos elaborando para verificar que expresen lo que deseamos y dialogamos con coautores y lectores intermedios sobre el texto para revisarlo. El texto final dependerá, en parte, de estas destrezas de comprensión lectora, oralización y conversación que posea el autor.

Sin embargo, en nuestra experiencia en formación de los docentes en lectura y escritura, este fue uno de los obstáculos iniciales por resolver, pues como docentes que no fuimos formados, ni en la escuela ni en la universidad, en estos objetos de conocimiento, presentábamos (y se presentan de forma permanente) grandes problemas al momento de escribir nuestros registros de clase; hoy continuamos construyendo este proceso.

Al asumir la investigación de las prácticas pedagógicas, tenemos que escribir para contar y sistematizar las experiencias didácticas. De tal modo, la exigencia de poner por escrito lo que se hace en el aula es tal vez lo que lleva a muchos docentes, que en un principio se incorporan entusiasmados a procesos de formación, a abandonar la capacitación, pues nadie está dispuesto a efectuar aquello que le resulta difícil, más bien tiende a evitarlo.

En el contexto de la lectura y la escritura, pensamos que los entes capacitadores como las universidades deben plantearse cómo hacer para que desde su ámbito los maestros y alumnos se mantengan activos, es decir, leyendo y escribiendo con un propósito definido. Estos planteamientos conducen a un interrogante ineludible: ¿cuáles estrategias son válidas para ayudar a los docentes a leer y a escribir?

Para conseguir alguna respuesta satisfactoria a esta pregunta, es necesario según Gutiérrez y Díez (2018) analizar las condiciones de trabajo del docente. Pues, a nuestro parecer, a un maestro que trabaja mañana, tarde y noche es casi imposible pedirle que escriba su historia, ya que si algo hemos 
construido en estos últimos años es que para escribir hay que sentarse, darse tiempo, elaborar borradores, tachar, quitar, poner; es como dijo Ribót (1997, p. 1): "cuestión de nalgas". Es importante que el maestro no esté solo en este camino, pues para construir hay que interactuar.

En tal sentido, debe ofrecerse a los docentes experiencias escritas por otros, que les ayuden a ampliar su visión del mundo, pues esto incidirá en su didáctica y en la reconstrucción de los esquemas propios como lector y escritor. Entonces, sostener que el conocimiento didáctico de la lectura y la escritura debe constituirse en el marco de la formación de los docentes, le otorga un lugar particular y supone, a la vez, la existencia de otros conocimientos en esta formación, y un modo de relacionarse con ellos.

Algunas cuestiones fundamentales para ayudar a los maestros a reconciliarse con la lengua escrita están relacionadas con la publicación y difusión de lo que escriben. Asimismo, crear espacios donde los profesores de primaria presenten sus experiencias innovadoras, es decir, darles sentido a las actividades de lectura y escritura. Para ello, es necesario convertir los espacios de capacitación en lugares donde la producción e interpretación de textos surjan como respuesta a las necesidades de los miembros del grupo. En este contexto, creamos espacios para la publicación de experiencias pedagógicas de los maestros: las revistas Maestros Hoy y LEID. ${ }^{2}$

La práctica del maestro como lector y escritor es de gran importancia para guiar el trabajo pedagógico como enseñante de lectura y escritura. Tal vez, esta cualidad es un indicador importante que influye de manera decisiva en el aprendizaje de los niños en relación con la adquisición y desarrollo de la lengua escrita. En consecuencia, si los encuentros y talleres de actualización que se propicien le permiten al docente relacionarse de forma efectiva con la lectura y la escritura, el problema de capacitación estaría prácticamente solucionado, pues utilizar la lengua escrita para mostrar los sucesos pedagógicos sería el camino por el cual debe transitar todo docente en la construcción de nuevos paradigmas didácticos que lo lleven a investigar su propia acción.

Uno de los aspectos que debe discutirse con los docentes, en sus procesos formativos, es lo relacionado con la enseñanza de la lectura y su comprensión.

2 Maestros Hoy es una revista donde los maestros tienen la posibilidad de contar sus experiencias. Dirigida por maestros para maestros difundida en Venezuela. Y LEID es un congreso sobre Lectura, Escritura, Investigación y Didáctica, también dirigido a los docentes, de todos los niveles y de cualquier país, que deseen dar a conocer sus experiencias.
Pues, es reconocido por los involucrados en la enseñanza, a decir de Ballester (2015), que las estrategias que contribuyen a la comprensión de la lectura para lograr un aprendizaje significativo no se utilizan con regularidad en el aula y el tiempo que los docentes dedican a leer allí es insuficiente. En este sentido, M. Pérez (2003) menciona que en el contexto educativo se ha visto la necesidad de enseñar a los estudiantes a comprender textos debido a la actitud de apatía, falta de interés, la dificultad para comprender un texto $y$, por ende, llegar a inferir a partir de la información que brinda una lectura o dar a conocer su punto de vista.

En efecto, los docentes en ejercicio saben que la enseñanza de la lectura y su comprensión es una de las capacidades lectoras de mayor complejidad. Pues, según M. J. Pérez (2005), en la escuela siempre se ha pensado que los niños son lectores pasivos, que la información se encuentra en el texto y el alumno tiene que extraerla. A esta etapa de extracción de la información, en esencia de palabras, se piensa que el alumno llega una vez que domina el proceso de decodificación de letras para componer palabras y, por ende, la comprensión de la lectura se deja para los niveles superiores.

Así, por ejemplo, en los niveles básicos de la escolaridad, los educadores enseñan primero las letras, luego a decodificar sílabas, de allí pasan a las palabras, y por último a leer oraciones. Además, las oraciones o frases se presentan a los escolares sin la discusión del significado, pues pareciera que la comprensión de los textos no es tarea de los grados iniciales. Este enfoque de la enseñanza de la lectura ha prevalecido por muchos años en los maestros de estos grados en los distintos países de Latinoamérica. En consecuencia (es nuestra opinión), si los gobiernos de los países latinoamericanos no desarrollan acciones que cambien el paradigma de la enseñanza de la lectura y la escritura, sus niveles de desarrollo se verán siempre afectados, pues la buena formación de lectores es un elemento para el desarrollo sostenible de cualquier nación.

Ahora bien, con los avances y aportes de la psicogenética y la psicolingüística, la concepción tradicional de la lectura, mencionada antes, ha presentado cambios teóricos importantes. El más significativo, según Goodman (1996), ha sido demostrar que el significado no está en el texto, está en el escritor cuando escribe y en la mente del lector cuando lee, por tanto, es un proceso totalmente activo de construcción de significados. En torno al cambio de la enseñanza de la lectura de la concepción tradicional a una que implique la participación del estudiante, es necesario generar acciones capacitadoras desde 
distintos entes relacionados con la educación. Con respecto a esta temática, vemos en la cotidianidad de las aulas que uno de los aspectos menos trabajado es la comprensión de la lectura.

En tal sentido, los planteamientos de Pérez y Roa (2010) reflejan la necesidad imperante que tienen los profesores acerca de desarrollos didácticos para caracterizar sus procesos de enseñanza de la comprensión lectora "en el campo del lenguaje conforme a los principales retos que demandan los estudiantes dadas sus características particulares, sus intereses, sus necesidades formativas" (p. 8).

Estos retos, que viven en su cotidianidad los docentes, deben llevarlos a buscar alguna formación como lector. Pues durante el proceso de enseñanza, desde transición hasta la educación superior, de acuerdo con Cerrillo (2016) se necesita leer una variedad de textos para apropiarse de diferentes conocimientos. La importancia de esto no solo reside en los contenidos, sino en la cantidad, propósitos y capacidad para comprender los textos. Como dice Dubois (2011), "No es una novedad afirmar que el elemento clave en el proceso educativo es la actuación del docente" (p. 66).

La mayoría de los docentes saben que, en gran medida, los aprendizajes que adquiere un estudiante le llegan a través de la lectura. Esto, dado que un estudiante con buena comprensión de lo que lee, es un estudiante exitoso. Por tanto, cuando en la escuela se habla de un alumno que comprende lo que lee, se está afirmando lo que dicen los expertos, como Smith (1990), para quien la comprensión lectora involucra la habilidad de comprender e interpretar una amplia variedad de tipos de texto y de dar sentido a lo leído al relacionarlo con los contextos en que aparecen. Este investigador de la lectura comprensiva afirmó que en el proceso de la lectura intervienen dos fuentes de información: la visual y la no visual. En la primera, se descodifica mediante la selección hecha por la vista y en la segunda, interviene la información que posee en su cerebro el lector. Ambas pueden intercambiarse; sin embargo, la segunda es indispensable en la comprensión y aprendizaje de la lectura.

En este sentido, Solé (2001) acota que la lectura constituye un acto dinámico entre el lector y el texto. Mediante el acto de la lectura, el lector busca satisfacer los objetivos que guían su acción y lo lleva a cabo a través de conexiones coherentes entre la información que posee en sus estructuras cognitivas y la nueva que suministra el texto. La competencia para com- prender los textos acompaña al ser humano durante toda la vida y representa una de las expresiones más significativas del conocimiento humano.

Es decir que, para disfrutar mejor de la vida, es necesario tener una buena comprensión de los textos. Y para ello la enseñanza debe permitir, como dicen Kaufman, Von, Marguery, Zaidenband y Maidana (2007), que los "alumnos participen en prácticas de lectura de diferentes tipos de textos de circulación social, con propósitos determinados, tal como se lee fuera del ámbito escolar" (p. 20).

En este orden de ideas, Solé (2001), afirma que:

La interpretación progresiva del texto, es decir, la elaboración de su comprensión implica determinar las ideas principales que contiene. Es importante establecer que, aunque un autor pueda confeccionar un texto para comunicar determinados contenidos, la idea o ideas principales que construye el lector dependen en buena parte de los objetivos que afronta la lectura, de sus conocimientos previos y de lo que el proceso de lectura en sí le ofrece en relación con los primeros. (p. 25).

De allí, que se diga, entonces, que las prácticas constantes de actividades lectoras se constituyen en entornos donde las personas acceden a información y la procesan para convertirla en conocimiento útil para la vida. Por ello, aprender a leer requiere de nuevas técnicas para la comprensión de textos. La misma Solé (1987) menciona que aprender a leer quiere decir que el ser humano debe comprender los mensajes impresos. Es decir, se requiere el dominio de destrezas lectoras cuya dificultad dependerá del propósito y tipo de texto.

Asimismo, Perdomo (2007) refiere que "la comprensión del texto se logra a través de las estrategias de lectura" (p. 63). Estas revelan la forma en que los lectores se desenvuelven en su interacción con el texto escrito, y cómo llegan a la comprensión de este. Entonces, la comprensión lectora debe ser vista como una habilidad de los seres humanos para procesar significado, además de ello, implica habilidades para el manejo de otros niveles de información lingüística como son el fonológico, el sintáctico y el pragmático. De igual manera, la lectura exige un diálogo secuencial entre lector y escritor.

Entonces, podría decirse que son muchos los aspectos que deben tomarse en consideración para contribuir con la formación de los docentes en lectura y escritura, pero lo más importante es buscar en ellos una reflexión permanente sobre sus prácticas como enseñantes y como escritores. 


\section{La relación escuela- universidad en el proceso de cambio en las prácticas pedagógicas de los docentes}

Si bien es cierto que todo profesional, y en particular los docentes, tenemos que actualizarnos y prepararnos cada día más, no es menos cierto que si la universidad cumpliera a cabalidad el papel de formadora de docentes críticos, participativos, lectores y productores de textos, el camino en la actualización tomaría otro rumbo. Pues, al docente que sale de la universidad con una alta formación en lectura y escritura, le resulta más fácil el camino de su actualización y capacitación permanente.

La realidad con que nos hemos encontrado en las aulas de clase, durante estos años de investigación, es que la gran mayoría de los docentes egresados de las diferentes universidades carecen de conceptos claros sobre lo que significa intelectual, social y afectivamente el acto lector y escritor. Dado que, de acuerdo con Lerner (1994), saber leer es comprender y saber escribir es producir texto, entonces si en el ejercicio de nuestra profesión no nos actualizamos en estos objetos de conocimiento, seguimos colaborando con la formación de un país de analfabetas funcionales.

Aquí se plantean algunas inquietudes producto de la discusión (como mencionamos al inicio de esta disertación) con estudiantes de pregrado y posgrado, $\mathrm{y}$ docentes en ejercicio:

- ¿Están conscientes las universidades del compromiso que tienen en la formación de los docentes como lectores y escritores?

- ¿Por qué el fracaso de la universidad en su función esencial de formación de un docente crítico, lector y productor de textos?

A este respecto, y en el contexto de la formación de docentes, Dubois (1993) sostiene que:

[...] la formación del docente debe contemplar, por un lado, la formación en el saber y como aplicarlo: y por otro, su desarrollo como persona; la formación en el ser [...] si el docente ha de hacer algo más que enseñar, si ha de educar en la lectura y la escritura: el mismo debe haber sido educado en tal sentido [...]. (p. 12).

La formación de un docente como lector y escritor, y como enseñante de lectura y escritura, es compromiso ineludible de las carreras de formación docente. Una universidad, según Díez y Clemente (2017), que estimule en el estudiante de educación el desarrollo de su capacidad como lector y escritor, está pensando en la formación de un docente para la vida, capaz de asumir nuevos retos en cuanto a su capacitación permanente.

Es importante que a los alumnos de las carreras de formación docente se les propicie actividades orientadas a brindar los conocimientos necesarios sobre el aprendizaje de la lengua escrita, de acuerdo con las teorías más recientes, para que reflexionen acerca de los principios fundamentales que debe orientar una propuesta didáctica basada en el respeto al aprendiz. De igual manera, los alumnos deben desarrollar actividades como lectores y productores de textos reales, y no solo con el único fin de aprobar asignaturas.

Ahora bien, ¿qué sucede con los docentes en ejercicio? ¿Cómo establecen las universidades una relación efectiva entre la escuela y el recinto universitario? En el contexto de la formación del docente, las universidades deben asumir la responsabilidad en la tarea de lograr mayores niveles de claridad sobre lo que es el proceso lecto-escritor, para que tanto los estudiantes como los docentes en servicio hagan uso efectivo de la lectura y la escritura como herramientas imprescindibles en la adquisición de todos los demás aprendizajes.

El compromiso de la universidad, en cualquier contexto, debe ser con la formación, capacitación y actualización del docente bajo una perspectiva renovadora que responda a las inquietudes mencionadas. En las siguientes experiencias, que se han venido poniendo en práctica en Venezuela con el concurso de algunas universidades, se sintetizan algunos de estos aspectos:

- Incorporación al currículo de la carrera de Educación un eje relacionado con la formación en oralidad, lectura y escritura

- Creación de programas de posgrado en Promoción de lectura y escritura

- Desarrollar programas de asistencia a los docentes en ejercicio

Las experiencias anteriores tienen que ver con lo que propone la universidad desde el interior hacia afuera. Pero esto no puede quedarse allí, también es necesario preguntarse: ¿Cómo desarrollar acciones para que los docentes en ejercicio se acerquen con sus alumnos a los contextos lectores? ¿Cómo la universidad propicia acciones para contribuir con la formación en el conocimiento didáctico de los educadores en ejercicio?

Pensar en procesos de formación centrados en el conocimiento didáctico, como dice Camps (1993), no supone eliminar otros aspectos de actualización docente. Es importante partir de las prácticas 
didácticas de los docentes e incluir prescripciones sobre cómo hacer en algunos momentos de la formación. También, reflexionar con otros, sobre la propia práctica, conocer las disciplinas de referencia (lectura y escritura) sobre el sujeto y el objeto de conocimiento. Es imprescindible vigilar la coherencia entre los aportes de estas disciplinas, consideradas relevantes para la formación de los niños, para hacer más conscientes las prácticas como lector y escritor. Sin embargo, en todos los casos, creemos que tales situaciones deben desarrollarse vinculadas con el propósito de ampliar conocimientos didácticos, en particular de la lectura y la escritura, que son en definitiva los objetos de conocimiento que potencian la investigación en el aula.

Estas acciones pueden quedarse a nivel macro y no incidir de forma positiva en la problemática planteada si cada uno de los docentes no asumimos el compromiso de cambio hacia nuevos paradigmas didácticos. En este sentido, durante dos lapsos de nuestra investigación (las aulas de clase para nosotros son espacios de investigación permanente) desarrollamos una experiencia de integración escuela-universidad en la asignatura Lectura y Escritura ${ }^{3}$ que, por un lado, ayudó a los estudiantes a conocer el contexto escolar y, por otro, contribuyó con los docentes en su proceso de cambio en las prácticas pedagógicas.

La primera parte de esta experiencia (en las dos universidades y en tiempos distintos) la dedicaron los estudiantes a observar la práctica de los docentes en sus aulas de clase, luego tuvieron un espacio para discutir y analizar lo observado, durante el cual estudiamos seis elementos: lectura, escritura, didáctica, aprendizaje, rol del docente y rol del alumno.

Después, los estudiantes seleccionaron dos niños con los cuales desarrollaron actividades de promoción de Lectura y Escritura. Para ejecutar estas actividades los alumnos conformaron equipos, de tal manera que mientras uno la llevaba a cabo, dos efectuaban el registro. Los registros de las actividades con los niños permiten el análisis de los estudiantes como lectores y productores de textos, y como futuros

3 Esta experiencia de integración escuela - universidad se efectuó en dos grandes momentos. El primer momento fue entre los años 1999-2001 con estudiantes de la carrera de Educación Integral de la Universidad de Los Andes en Venezuela, cuyos estudiantes desarrollaron un trabajo de promoción de lectura con alumnos de educación primaria. El segundo momento fue en el año 2017 (periodos 1 y 2), donde los estudiantes de la carrera de Educación Integral de la Universidad Nacional Abierta de la Unidad de Apoyo Coloncito efectuaron un trabajo de acompañamiento en lectura y escritura con estudiantes de Educación Primaria, como parte de sus Prácticas profesionales. De estas dos experiencias se extraen reflexiones para este artículo por ser las más significativas. enseñantes de Lectura y Escritura. Durante la discusión se hizo revisión de los aspectos teóricos de estos objetos de conocimiento.

Luego de avanzar en el trabajo con los niños elegidos, los estudiantes desarrollaron una práctica en una escuela seleccionada por ellos con todos los niños de una sección. Allí escribieron el registro de la actividad para luego sistematizar la experiencia y darla a conocer: de tal modo se hace Investigación Acción desde la práctica. Es interesante mencionar que durante las sesiones con los alumnos de una escuela surgieron distintos proyectos, entre ellos se destaca un intercambio de correspondencia entre los alumnos de la Universidad de Los Andes y los niños de una de las escuelas visitadas, actividad que fue altamente significativa para hacer de la escritura un proceso funcional y con sentido en ambos contextos.

Otra fase de esta experiencia tiene que ver con la publicación y difusión del trabajo desarrollado por los estudiantes y/o maestros, con el fin de que este no se quede solo como un requisito para aprobar las asignaturas o para cumplir instrucciones. Pues, hoy creemos que el contacto con el mundo les da una experiencia bien interesante a los futuros educadores. Al respecto, se elaboran informes y ensayos para ponencias con el fin de participar en eventos relacionados con la docencia. Entre algunos de estos eventos, que hemos fundado tanto para los estudiantes como para los maestros, se destacan el Seminario Lectura, Escritura y Formación Docente, Seminario de investigación cualitativa, y Congreso Nacional LEID - Lectura, Escritura, Investigación y Didáctica.

Entonces, podemos decir que en nuestra propuesta los procesos de formación docente, que saltan desde las investigaciones efectuadas, tienen como objetivo: a) crear oportunidades para que los estudiantes asuman la lectura y la escritura como actos de vida y no para preparar temas para un examen, o para tomar apuntes de clase; b) ensayar una práctica pedagógica que apunte al desarrollo de los alumnos como lectores y productores de textos. Una práctica que se convierta en uno de los espacios más significativos para la formación permanente y c) establecer un puente para que los docentes en ejercicio se relacionen con las propuestas pedagógicas innovadoras planteadas desde la Universidad. 


\section{Algunas consideraciones finales}

El análisis de los tres planteamientos mencionados conduce a algunas consideraciones generales:

- La construcción de un nuevo rol docente que parta de reflexiones sobre la lectura y la escritura se convierte en un tema que debe ocupar un lugar y un tiempo de análisis en todas las instituciones de formación de maestros. Cuando mencionamos UN NUEVo ROL DOCENTE, nos referimos, así en mayúsculas, al docente que abandona el modelo educativo como traspaso de información y se inserta en prácticas renovadoras que respeten el proceso del aprendiz.

- La lectura y la escritura pasan a ocupar líneas de trabajo imprescindibles en un docente que investiga sobre su propia acción.

- La tarea pedagógica se convierte en atractiva cuando parte de una búsqueda permanente, cuando se presentan nuevos retos, cuando se integra la teoría con la práctica, cuando nos damos la posibilidad de dar y recibir, de aprender de nosotros y de los otros, es decir, cuando le damos sentido a lo que hacemos.

- No se puede esperar un docente transformado como por arte de magia, esto lleva tiempo y esfuerzo. Se necesita tanto del concurso de todas las instancias involucradas en la formación docente como del aporte individual, que viene a ser el más importante.

- Es imprescindible que la progresión de los conocimientos relacionados con la lectura y la escritura que se decida presentar a los niños parta de un proceso de selección realizado en función de determinados propósitos. A fin de cuentas, son los saberes más utilizados en el aula, por lo cual hay que diseñar situaciones didácticas para comunicarlos de tal manera que sean útiles para la vida.

- Otro aspecto importante por revisar está relacionado con los tipos de intervenciones que el docente debe hacer durante el desarrollo de las situaciones didácticas, en especial, ante los "errores" de los alumnos. Pues estos "errores" se constituyen en la mayor fuente de aprendizaje.

- Aunque no existe una fórmula mágica (pues así lo hemos observado en nuestras investigaciones), es necesario revisar la manera más adecuada para organizar la clase y distribuir roles en cada situación de lectura y de escritura, que permita la construcción de conocimientos en los alumnos.

- Por último, consideramos que, si bien es cierto que el conocimiento didáctico se construye elaborando situaciones de enseñanza y poniéndolas a prueba en diversos contextos, no es menos cierto que las situaciones de formación de docentes en servicio y de los estudiantes de docencia constituyen una fuente permanente de conceptualizaciones de nuevos problemas didácticos. A nuestro juicio, esta fuente es casi tan importante como los procesos desarrollados en el aula con los niños, lo cual podría convertir las aulas de clase en verdaderos laboratorios para la investigación.

\section{Referencias}

Ballester, J. (2015). La formación lectora y literaria. Barcelona: Graó.

Camps, A. (1993). Didáctica de la lengua: la emergencia de un campo científico específico. Infancia y Aprendizaje, 62/63, 209-217.

Camps, A. (coord.) (2001). El aula como espacio de investigación y reflexión. Barcelona: Graó.

Cassany, D. (1999). Construir la escritura. Barcelona: Paidós.

Cassany, D. (2003). Describir el escribir, cómo se aprende a escribir. Barcelona: Paidós.

Castedo, M. (directora). (2010). Lectura y escritura en la educación rural. Proyecto de extensión. Universidad Nacional de La Plata. Facultad de Humanidades y Ciencias de la Educación. Recuperado de http://www. memoria.fahce.unlp.edu.ar/extension/px.50/px.50.pd

Cerrillo, P. C. (2016). El lector literario. México: Fondo de Cultura Económica.

Díaz, M. (2016). La investigación y la didáctica de la historieta, como herramienta de aprendizaje en la enseñanza de adultos. Opción, 32, [No. especial 7]. 589-582, Recuperado de http://www.redalyc.org/ html/310/31048480033/

Díez, A. y Clemente, V. (2017). Didáctica de la lengua y la literatura para los estudiantes para maestro/a: Saberes previos y reflexión didáctica. En R. Roig-Vila (Ed.), Investigación en docencia universitaria. Diseñando el futuro a partir de la innovación educativa (pp. 180-196). Barcelona: Octaedro.

Dubois, M. (1993). Formación del docente como lector. Lectura y vida, 4, 3-10, diciembre. 
Dubois, M. (1995). Lectura, escritura y formación docente. Lectura y vida, 2, 5-11, febrero.

Dubois, M. (2011). La lectura en la formación y actualización del docente. Comentario sobre dos experiencias. Legenda, 15 (12), 65-79. Recuperado de http://erevistas.saber.ula.ve/index.php/legenda/article/view/733

Goodman, K. (1996). La lectura, la escritura y los textos escritos: Una perspectiva transaccional sociopsicolingüística. En Textos en contexto II. Lectura y Vida, (pp. 11-68). Argentina: Asociación Internacional de Lectura.

Gutiérrez, R. y Díez, A. (2018). Conciencia fonológica y desarrollo evolutivo de la escritura en las primeras edades. Educación XX1, 21 (1), 395-416. Recuperado de http:// www. article/view/73310.5944/educXX1.13256

Gutiérrez, R. y Molina, R. (2017). ¿Qué saben los futuros docentes sobre el proceso inicial del aprendizaje del lenguaje escrito? En R. Roig-Vila (Ed.), Investigación en docencia universitaria. Diseñando el futuro a partir de la innovación educativa (pp. 78-90). Barcelona: Octaedro.

Jiménez, E. (2018). La inteligencia emocional como predictor del hábito lector y la competencia lectora en universitarios. Investigaciones sobre lectura, 10, 30-54. Recuperado de https://www.comprensionlectora.es/ revistaisl/index.php/revistaISL/article/view/256/109

Kaufman, A., Von, C., Marguery, M., Zaidenband, A. y Maidana, J. (2007). Leer y escribir en el día a día en las aulas. Buenos Aires: Aique.

Lerner, D. (1994). Capacitación en servicio y cambio en la propuesta didáctica vigente. Lectura y vida, 15 (3), 33-54.

Perdomo, B. (2007). Estrategias de lectura usadas por los estudiantes de nuevo ingreso en la Facultad de Odontología de la Universidad de Los Andes, Venezuela. Recuperado de http://kaleidoscopio.uneg.edu.ve/numeros/ k08/k08_art06.pdf
Pérez, M. (2003). Leer y escribir en la escuela: algunos escenarios pedagógicos y didácticos para la reflexión. Bogotá: ICFES. Recuperado de http://cms.univalle. edu.co/todosaaprender/anexos/enelcamino/2-MENLeeryescribirenlaescuela.pdf

Pérez, M. J. (2005). Evaluación de la comprensión lectora: dificultades y limitaciones [Núm. Extraordinario]. Revista de Educación, pp. 121-138. Recuperado de http://www.ince.mec.es/revistaeducacion/re2005/ re2005_10.pdf.

Pérez, M. y Roa, C. (2010). Referentes para la didáctica del lenguaje en el primer ciclo. Bogotá: Secretaría de Educación del Distrito.

Ribót, S. (1997). Escribir en la escuela [Mimeografiado]. Valencia.

Salas, P. (2012). El desarrollo de la comprensión lectora en los estudiantes del tercer semestre del nivel medio superior de la Universidad Autónoma de Nuevo León. México. Recuperado de http://eprints.uanl. $\mathrm{mx} / 3230 / 1 / 1080256466 . p d f$

Silva, M. (2014). El estudio de la comprensión lectora en Latinoamérica: necesidad de un enfoque en la comprensión. Innovación Educativa, 14 (64), 47-56.

Smith, F. (1990). Para darle sentido a la lectura. Madrid: Aprendizaje Visor.

Solé, I. (1987). Las posibilidades de un modelo teórico para la enseñanza de la comprensión lectora. Infancia y Aprendizaje. Recuperado de https://dialnet.unirioja. es/descarga/articulo/749227.pdf

Solé, I. (2001). Estrategias de lectura. Barcelona: Graó. 